\title{
Are gamma rays produced in the core region of microquasars and AGNs?
}

\author{
Behrouz Khiali* \\ University of Sao Paulo) \\ E-mail: bkhialieusp
}

Elisabete de Gouveia Dal Pino

University of Sao Paulo

E-mail: dalpinodiag.usp.br

Maria V. del Valle

IAR, CONICET, Argentina

E-mail: mariaeiar-conicet.gov.ar

Helene Sol

Observatoire de Meudon, France

E-mail: Helene. Soldobspm. fr

\begin{abstract}
Cosmic Ray (CR) acceleration is still challenging in high energy astrophysics. A first-order Fermi mechanism within magnetic reconnection layers has been demonstrated to be a powerful CR accelerator in recent studies. In this work we have investigated this acceleration process in the nuclear region of radio-galaxies and microquasars and found that the very high energy (VHE) emission from these astrophysical sources may be originated in the nuclear region around the central black hole. We employed both lepontic and hadronic models to interpret the observed gamma emission resulting from interactions of accelerated particles by magnetic reconnection with the ambient radiation, magnetic and matter fields. We compared the acceleration rate with the proper cooling rates obtaining the maximum particle energy and then reconstructed the spectral energy distribution (SED) for a few galactic and extragalactic sources and found that the are consistent with the observations. Also this model naturally explains the fast time variability of the emission of these sources.
\end{abstract}

The 34th International Cosmic Ray Conference,

30 July- 6 August, 2015

The Hague, The Netherlands

${ }^{*}$ Speaker. 


\section{Introduction}

There are many open questions regarding the origin of CRs and their properties which are directly related to astrophysical issues and most intriguing puzzles of modern physics, such as the nature of Dark Matter.

In particular, the acceleration and propagation of CRs are key processes in the understanding of the high energy, non-thermal universe. They are produced everywhere in the cosmos: in compact sources, like pulsars, supernova remnants, galactic black hole binaries (or microquasars), active galactic nuclei (AGNs) and gamma ray bursts (GRBs), as well as in more diffuse media, like in shocked regions of the intracluster gas. Understanding how they are produced in these different environments and how they propagate through the intergalactic and intracluster turbulent magnetized medium is still under debate.

These open questions require a multi-messenger approach to be solved. It is important to look not only for the CR particles, themselves, but also at the radiation they produce through various mechanism such as synchrotron, Bremmstrahlung, Inverse Compton, decay pion decay via $p p$ inelastic collisions and $p \gamma$ interactions. In particular, Gamma-ray observations of the regions surrounding astrophysical sources are an important tool to constrain the diffusive behavior of CRs. This is because CRs after leaving their sources produce gamma rays in interactions with the ambient gas, radiation and magnetic fields surrounding the accelerator.

In this work, microquasars and low luminosity AGNs (LLAGNs) are our main target to study. We consider magnetic reconnection model in which particles are accelerated, through a first-order Fermi process, in the surrounds of the $\mathrm{BH}$ by the magnetic power extracted from fast magnetic reconnection events occurring between the magnetosphere of the $\mathrm{BH}$ and the magnetic field lines arising from the inner accretion disk. Inspired by similar phenomena occurring in space environments, like the earth magnetotail and the solar corona, [3] (hereafter GL05) explored this process first in the framework of microquasars and then [4] (hereafter GPK10) extended it to AGNs.

In order to reconstruct the SED, we consider the relevant radiative processes due to the interactions of the accelerated particles by magnetic reconnection with the surrounding radiation, matter and magnetic fields. We then compare the rates of these radiative losses with the magnetic reconnection acceleration rate and determine the maximum energy that the electrons and protons can attain. For comparison, we also consider the acceleration rate due to shocks in the surrounds of the $\mathrm{BH}$, but find that this is less effective than the acceleration by magnetic reconnection.

\section{Our Scenario}

In our model, relativistic particles may be accelerated in the nuclear region of $\mu \mathrm{QSRs}$ ad LLAGNs, i.e., in the surrounds of the BH. This may happen as a result of fast magnetic reconnection and examine whether this process may reproduce the observed flares specially at VHEs. A brief description has been presented below (See $[3,5]$ for more details).

We assume that the inner region of the accretion disk/corona system alternates between two states which are controlled by changes in the global magnetic field. Right before a fast magnetic reconnection event, we adopt the simplest possible configuration by considering a magnetized standard geometrically thin and optically thick accretion disk around the BH. 


\subsection{Fast reconnection in the surrounds of the $\mathrm{BH}$}

The magnetosphere around the central $\mathrm{BH}$ can be built from the drag of magnetic field lines by the accretion disk. The large-scale poloidal magnetic field in the disk corona can in turn be formed by the action of a dynamo inside the accretion disk or dragged from the surroundings. Under the action of disk differential rotation, this poloidal magnetic flux gives rise to a wind that partially removes angular momentum from the system and increases the accretion rate. This, in turn, increases the ram pressure of the accreting material that will press the magnetic lines in the inner disk region against the lines of the $\mathrm{BH}$ magnetosphere thus favouring the occurrence of reconnection. We note that according to mean field dynamo theory, an inversion of the polarization of the magnetic lines is expected to occur every half of the dynamo cycle; when this happens a new flux of disk lines should reach the inner region with an inverted polarity with respect to the magnetic flux already sitting around the $\mathrm{BH}$, therefore, favouring magnetic reconnection between the two fluxes. The advection of field lines from the outer regions also allows for periodic changes in the polarity (see more details in KGS15).

The fluids we are investigating here have large hydrodynamical and magnetic Reynolds numbers implying that they can be easily distorted and become turbulent. According to the LV99 model, even weak embedded turbulence causes the wandering of the magnetic field lines which allows for many independent patches to reconnect simultaneously making the global reconnection rate large, $V_{R} \sim v_{A}\left(l_{i n j} / L\right)^{1 / 2}\left(v_{\text {turb }} / v_{A}\right)^{2}$, where $V_{R}$ is the reconnection speed, $v_{A}$ is the Alfvén speed, and $l_{\text {inj }}$ and $v_{\text {turb }}$ the injection scale and velocity of the turbulence, respectively. This expression indicates that the reconnection rate can be as large as $\sim V_{A}$. We should also notice that there has been direct evidences of turbulent reconnection in solar coronal eventsand also in the Earth magnetotail.

The physical quantities of magnetic reconnection model such as magnetic field (B), coronal number density $\left(n_{c}\right)$, released magnetic reconnection power by turbulent driven fast reconnection (W), disc temperature $\left(T_{d}\right)$ and the thickness of reconnection layer in the magnetic discontinuity region $\left(\Delta R_{X}\right)$ has been derived in KGS15 and applied here.

\subsection{Magnetic reconnection as a CR accelerator}

A first-order Fermi acceleration may occur when particles of the fluid are trapped between the two converging magnetic flux tubes moving to each other in the magnetic reconnection discontinuity with a velocity $V_{R}$. GL05 first investigated this process analytically and showed that, as the particles bounce back and forth undergoing head-on collisions with magnetic fluctuations in the current sheet, their energy increases by $\left\langle\Delta E / E>\sim 8 V_{R} / 3 c\right.$ after each round trip, which leads to an exponential energy growth after several round trips. If magnetic reconnection is fast, $V_{R}$ is of the order of the local Alfvén speed $V_{A}$ and, at the surroundings of relativistic sources $V_{R} \simeq v_{A} \simeq c$ and thus the mechanism can be rather efficient.

From the results of 3D MHD numerical simulations of this process [8], we find that the acceleration rate for a proton is given by $(\mathrm{KGV} 15)$ :

$$
t_{a c c, r e c, p}^{-1}=1.3 \times 10^{5}\left(\frac{E}{E_{0}}\right)^{-0.4} t_{0}^{-1},
$$

where $E$ is the energy of the accelerated proton, $E_{0}=m_{p} c^{2}, m_{p}$ is the proton rest mass, $t_{0}=l_{a c c} / v_{A}$ is the Alfvén time, and $l_{a c c}$ is the length scale of the acceleration region. 
Similarly, for the electrons one can get:

$$
t_{\text {acc }, \text { rec }, e}^{-1}=1.3 \times 10^{5} \sqrt{\frac{m_{p}}{m_{e}}}\left(\frac{E}{E_{0}}\right)^{-0.4} t_{0}^{-1},
$$

where $m_{e}$ is the electron rest mass.

The two equations above do not take into account the effects of radiative losses upon the accelerated particles. They will be used to compute the acceleration rates in our model.

As stressed in GL05, it is also possible that a diffusive shock may develop in the surrounds of the magnetic reconnection zone at the jet launching region caused by plasmons or coronal mass ejections. As in solar flares, these can be produced in the reconnection layer and released along the magnetic field lines. In this case, we expect the shock velocity to be predominantly parallel to the magnetic lines. and the acceleration rate for a particle of energy $E$ will be approximately given by:

$$
t_{\text {acc }, \text { shock }}^{-1}=\frac{\eta e c B}{E},
$$

where $0<\eta \ll 1$ characterizes the efficiency of the acceleration. We fix $\eta=10^{-1}$, which is appropriate for shocks with velocity $v_{s} \approx 0.1 c$ commonly assumed in the Bohm regime.

\subsection{Radiative cooling processes and absorption}

We assumed both leptonic and hadronic radiative loss mechanisms in the emission region. Emission region for $\mu \mathrm{QSR}$ has been considered as a core region around central $\mathrm{BH}$ and for LLAGNs corresponds to the torus with volume $V$ that in both cases encompass the cylindrical shell where magnetic reconnection particle acceleration takes place (For more details see [6,7]).

Accelerated particles due to reconnection mechanism may interact with the surrounding magnetic, charged matter, and photon fields.

Accelerated electrons and protons spiralling in the magnetic field emit synchrotron radiation. We calculate the synchrotron loss rate for the sources considered here and the radiated synchrotron spectrum as functions of the scattered photon energy. Electron interactions with the electrostatic field of nuclei of charge Ze allow for the production of bremsstrahlung radiation. Finally, relativistic electron interactions with photons may produce inverse Compton (IC) radiation.

Accelerated protons produce hadronic emission from interactions with the magnetic field (synchrotron), and also through the decay of neutral pions via $p p$ and $p \gamma$ interactions. A single pion can be produced in an interaction near the threshold and then decay giving rise to gamma-rays.

The cooling rates due to these leptonic and hadronic mechanisms are plotted as functions of the particle energy for the sources here studied in next section where they are also compared with the acceleration rates due to shock and magnetic reconnection.

The produced $\gamma$-rays can be annihilated by the surrounding radiation field via electron-positron pair production, i.e., $\gamma+\gamma \rightarrow e^{+}+e^{-}$. In our model the dominant radiation field for this process in the coronal region is due to the scattered photons from the accretion disk. The attenuated $\gamma$-ray luminosity $L_{\gamma}\left(E_{\gamma}\right)$ at a distance $z$ above the disk is given by

$$
L_{\gamma}\left(E_{\gamma}\right)=L_{\gamma}^{0}\left(E_{\gamma}\right) e^{-\tau\left(z, E_{\gamma}\right)}
$$

where $L_{\gamma}^{0}$ is the intrinsic coronal gamma-ray luminosity and $\tau\left(z, E_{\gamma}\right)$ is the optical depth. The calculated optical depth depends on the $\gamma$-ray energy and the distance above the disk $z$. 
Table 1: Model parameters for Cyg X-1 and M87.

\begin{tabular}{llrr}
\hline & Parameters & Cyg X-1 & M 87 \\
\hline$B$ & Magnetic field (G) & $2.3 \times 10^{7}$ & 1620 \\
$W$ & Magnetic reconnection power (erg/s) & $3.6 \times 10^{36}$ & $5.25 \times 10^{44}$ \\
$\Delta R_{X}$ & Width of the current sheet $(\mathrm{cm})$ & $1.1 \times 10^{7}$ & $1.35 \times 10^{15}$ \\
$n_{c}$ & Coronal particle number density $\left(\mathrm{cm}^{-3}\right)$ & $4.5 \times 10^{16}$ & $3.3 \times 10^{8}$ \\
$T_{d}$ & Temperature of the disk $(\mathrm{K})$ & $4.4 \times 10^{7}$ & $5.2 \times 10^{8}$ \\
$R_{x}$ & Inner radius of disk $(\mathrm{cm})$ & $2.6 \times 10^{7}$ & $5.3 \times 10^{15}$ \\
$L_{X}$ & Extension of the reconnection region $(\mathrm{cm})$ & $4.3 \times 10^{7}$ & $4.4 \times 10^{15}$ \\
$L$ & Extension of the corona $(\mathrm{cm})$ & $4.3 \times 10^{7}$ & $4.4 \times 10^{15}$ \\
$V$ & Volume of emission region $\left(\mathrm{cm}^{3}\right)$ & $3.5 \times 10^{2} 3$ & $10^{48}$ \\
$d$ & Distance of the source $(\mathrm{Mpc})$ & $2 \times 10^{-3}$ & 16.7 \\
$m$ & Mass of BH $\left(M_{\odot}\right)$ & 14.8 & $3 \times 10^{9}$ \\
$p$ & Injection spectral index & 1.8 & 2.4 \\
$\gamma_{\min }$ & Particle minimum Lorentz factor & 2 & 4 \\
\hline & & &
\end{tabular}

\section{Applications to astrophysical sources}

Here we show our results on applications of the model described above to $\mu$ QSRs (Cygnus X-1 as an example) and LLAGNs (e.g., M87). More sources of each astrophysical sources has been applied (see [6,7]), but here we show only one example of each classes. Physical parameters calculated by model nad observation listed in Table 1 .

\subsection{Application to Cygnus X-1}

Cyg X-1 is a widely studied black hole binary system at a distance of 1.86-2.2 kpc which is accreting from a high mass companion star orbiting around the $\mathrm{BH}$ with a period is 5.6 days. The orbit inclination is between $25^{\circ}$ and $35^{\circ}$ with an eccentricity of $\sim 0.018$, so that one can assume an approximate circular orbit with a radius $r_{\text {orb }}$.

The parameters of the model for Cyg X-1 are tabulated in Table 1. The volume $V$ of the emission region in Table 1 was calculated by considering the spherical region that encompasses the reconnection region in Figure 1.

Figures 2 and 3 show the cooling rates for the different energy loss processes described in Section 2.3 for electrons and protons. These are compared with the acceleration rates due to firstorder Fermi acceleration by magnetic reconnection and to shock acceleration.

We notice that for both protons and electrons the acceleration is dominated by the first-order Fermi magnetic reconnection process in the core region. The intercept between the magnetic reconnection acceleration rate and the synchrotron rate in Figure 1(a) gives the maximum energy that the electrons can attain in this acceleration process, which is $\sim 10 \mathrm{GeV}$. Protons on the other hand, do not cool as efficiently as the electrons and can attain energies as high as $\sim 4 \times 10^{15} \mathrm{eV}$ (Fig. 1(b)).

In order to reproduce the observed SED, we have calculated the non-thermal emission processes as described in Section 2.3 in the surrounds of the BH. Figure 2 shows the computed SED 


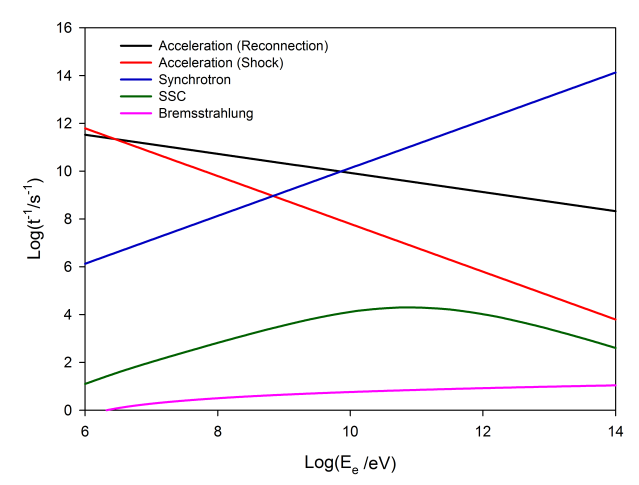

(a)

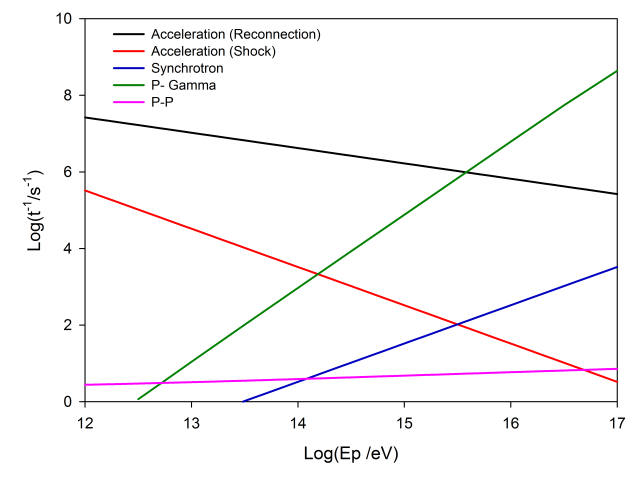

(b)

Figure 1: Acceleration and cooling rates for electrons (a) and for protons (b) in the nuclear region of Cyg $\mathrm{X}-1$.

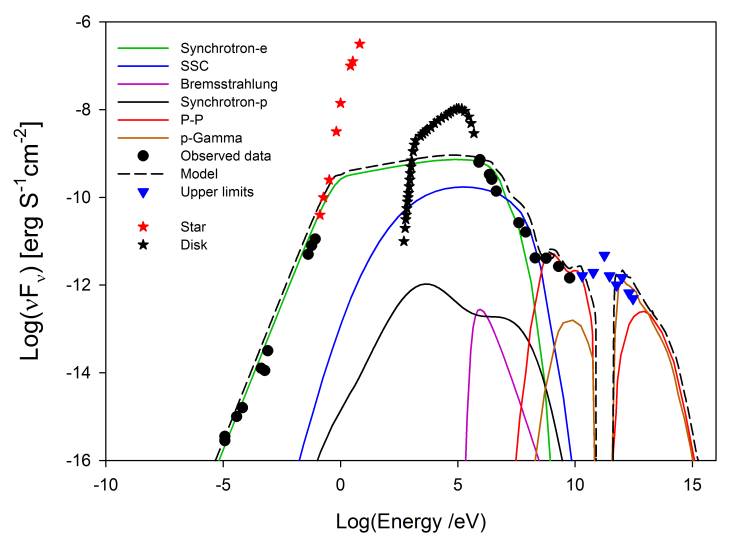

Figure 2: Calculated spectral energy distribution for Cyg X-1 using the magnetic reconnection acceleration model compared with observations (Extrcted from [6]).

for Cyg X-1 compared with observed data. As remarked, we have also considered the gamma-ray absorption due to electron-positron pair production resulting from interactions of the gamma-ray emission in the core with the surrounding radiation field. As stressed, our calculations indicate that this process is dominated by the radiation field of the companion star.

The calculated opacity according to the equations above results in a very high energy gamma ray absorption. We find that the produced gamma-rays are fully absorbed in the energy range of 50 Gev-0.5 TeV which causes the energy gap seen in the calculated SED in Figure 2. The observed upper limits by MAGIC plotted in the diagram in this range are possibly originated outside the core, along the jet where $\gamma$-ray absorption by the stellar radiation is not important.

The observed emission in the near infrared $(0.1 \mathrm{eV}-10 \mathrm{eV})$, represented in Figure 4 by red stars is attributed to thermal blackbody radiation from the stellar companion, and the accretion $\mathrm{X}$-ray emission ( $1 \mathrm{keV}-0.1 \mathrm{MeV}$ ) also represented in Figure 4 by dark stars, is believed to be due to thermal Comptonization of the disk emission by the surrounding coronal plasma of temperature 


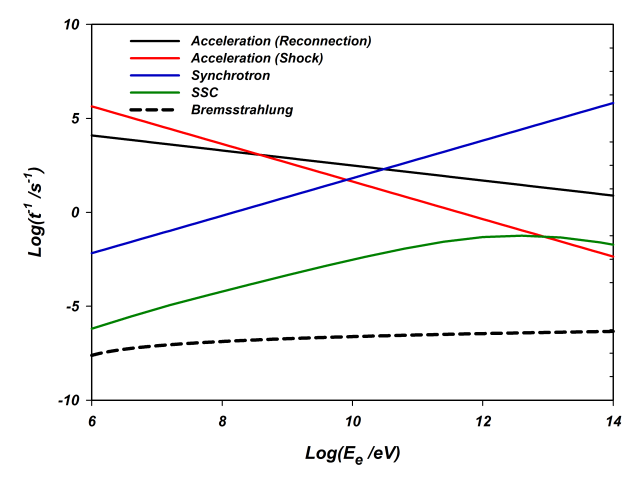

(a)

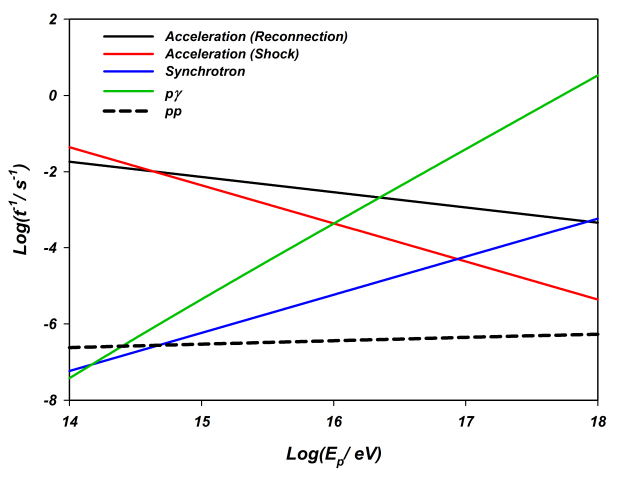

(b)

Figure 3: Acceleration and cooling rates for electrons (a) and for protons (b) in the nuclear region of M87.

$\sim 10^{7} \mathrm{~K}$. For this reason, these observed data are not fitted by the coronal non-thermal emission model investigated here.

\subsection{Application to M87}

The FR I giant radiogalaxy M87 is another well-known nearby AGN located at $16.7 \mathrm{Mpc}$ within the Virgo cluster which harbours a SMBH with a mass of $M_{B H} \sim 6 \times 10^{9} M_{\odot}$. The TeV $\gamma$-ray signal from M87 was first reported by HEGRA ([1]) and then confirmed by HESS ([2]). The latter also revealed that this emission is strongly variable with time scales of 1-2 days and thus produced in a very compact region, as pointed out before.

Table 1 shows the parameters that we used to calculate the acceleration and cooling rates and also to reconstruct the SED of this source.

In Figures 3, we compare the rates of the radiative cooling processes with the rates of the acceleration mechanisms for electrons and protons. We find that the dominant energy loss mechanisms are the synchrotron and the $p \gamma$ interactions for electrons and protons, respectively, and the acceleration is dominated by the magnetic reconnection process which defines the energy cut off for both electrons and protons. Figure 3(a) indicates that this maximum energy is $\sim 4 \times 10^{10} \mathrm{eV}$ for electrons and $\sim 5 \times 10^{16} \mathrm{eV}$ for protons.

Figure 4 shows the calculated SED for M87 compared to the observations.

The spectrum detected by the Fermi-LAT connects smoothly with the low-state $\mathrm{TeV}$ tail detected by HESS. In Figure 4, our model reproduces this connection effectively with $\gamma$-ray emission produced by SSC emission and $p p$ collisions. The observed $\mathrm{TeV}$ tail by HESS is fitted by the decay of neutral pions from $p \gamma$ interactions.

We note that our model and the chosen parametrization is also consistent with the observed $\mathrm{TeV}$ rapid variability of M87 which is $\sim 1-2$ days implying an extremely compact emission region (corresponding to scales of only a few $R_{S}$ ). 


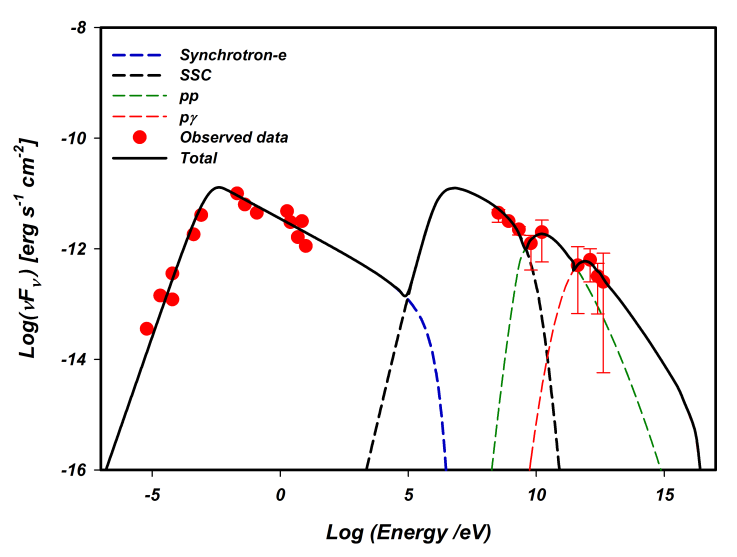

Figure 4: A lepton-hadronic model of the SED of M 87 compared with observations (Extracted from [7]).

\section{Conclusions}

- We have presented a reconnection acceleration model in the core region around the BH of the LLAGNs and $\mu$ QSRs and showed that it is able to reproduced very well their SEDs, from radio to gamma-rays up to $\mathrm{TeV}$ energies.

- Magnetic reconnection acceleration seems to provide a better efficiency in regions where magnetic activity is dominant in comparison with diffusive shock acceleration as the cores of LLAGNs and $\mu$ QSRs. Particles can gain energy up to a few times $\sim 100 \mathrm{PeV}$ due to magnetic reconnection acceleration.

- The observed $\mathrm{TeV} \gamma$-ray emission may be originated in these cores via neutral pion decays in hadronic processes.

- The fast magnetic reconnection acceleration model occurring in the core of these sources can naturally explain the observed short time variability, specially of the high energy $\gamma$-ray.

\section{References}

[1] Aharonian, F. A., Akhperjanian, A., Beilicke, M., et al. 2003, A\&A, 403, L1

[2] Aharonian, F. A., et al. 2006, Science, 314, 1424

[3] de Gouveia Dal Pino, E. M. \& Lazarian, A. 2005, Astronomy and Astrophysics, 441, 845 (GL05)

[4] de Gouveia Dal Pino, E. M., Piovezan, P. P. \& Kadowaki, L. H. S. 2010a, Astronomy and Astrophysics, 518, A5

[5] Kadowaki, L. H. S., de Gouveia Dal Pino, E. M., \& Singh, C. B. 2015, ApJ, 802, 113

[6] Khiali, B., de Gouveia Dal Pino, E. M. \& del Valle, M. V. 2015a, MNRAS, 449, 34 (KGV15)

[7] Khiali, B., de Gouveia Dal Pino, E. M. \& Sol, H. 2015b, arXiv:1504.07592 (KGS15)

[8] Kowal, G., de Gouveia Dal Pino, E. M., Lazarian, A. 2012, Physical Review Letters, 108, 241102 